\title{
Universiteit
}

Leiden

The Netherlands

\section{Fear of Falling After Hip Fracture: A Systematic Review of Measurement Instruments, Prevalence, Interventions, and Related Factors}

Visschedijk, J.; Achterberg, W.; Balen, R. van; Hertogh, C.

\section{Citation}

Visschedijk, J., Achterberg, W., Balen, R. van, \& Hertogh, C. (2010). Fear of Falling After Hip Fracture: A Systematic Review of Measurement Instruments, Prevalence, Interventions, and Related Factors. Journal Of The American Geriatrics Society, 58(9), 1739-1748. doi:10.1111/j.1532-5415.2010.03036.x

Version: $\quad$ Not Applicable (or Unknown)

License: $\quad$ Leiden University Non-exclusive license

Downloaded from: https://hdl.handle.net/1887/117596

Note: To cite this publication please use the final published version (if applicable). 


\title{
Fear of Falling After Hip Fracture: A Systematic Review of Measurement Instruments, Prevalence, Interventions, and Related Factors
}

\author{
Jan Visschedijk, MD, MPH, ${ }^{* \dagger}$ Wilco Achterberg, MD, PhD, ${ }^{*}$ Romke van Balen, MD, PhD, ${ }^{\ddagger}$ and \\ Cees Hertogh, MD, PhD*
}

The objective of this review was to systematically describe and analyze fear of falling $(\mathrm{FoF})$ in patients after a hip fracture, focusing on measurement instruments for FoF, the prevalence of FoF, factors associated with FoF, and interventions that may reduce FoF. Fifteen relevant studies were found through a systematic literature review, in which the PubMed, Embase, PsychINFO, and CINAHL databases were searched. Some of these studies indicated that $50 \%$ or more of patients with a hip fracture suffer from FoF, although adequate instruments still have to be validated for this specific group. FoF was associated with several negative rehabilitation outcomes, such as loss of mobility, institutionalization, and mortality. FoF was also related to less time spent on exercise and an increase in falls, although knowledge about risk factors, the prevalence over a longer time period, and the exact causal relations with important health outcomes is limited. Most studies suffer from selection bias by excluding patients with physical and cognitive disorders. Hence, more research is required, including in patients who are frail and have comorbidities. Only when knowledge such as this becomes available can interventions be implemented to address FoF and improve rehabilitation outcomes after a hip fracture. J Am Geriatr Soc 58:17391748, 2010.

Key words: hip fractures; rehabilitation; fear of falling; falls efficacy; elderly

From the ${ }^{*}$ Department of Nursing Home Medicine Amsterdam, VU University Medical Centre/EMGO Institute for Research in Extramural Medicine, Amsterdam, the Netherlands; ${ }^{\dagger}$ Zorggroep Solis, Deventer, the Netherlands; and ${ }^{\ddagger}$ Department of Public Health and Primary Care, Leiden University Medical Centre, Leiden, the Netherlands.

Address correspondence to Jan Visschedijk, Department of Nursing Home Medicine Amsterdam, VU University Medical Centre/EMGO Institute for Research in Extramural Medicine, Van der Boechorststraat 7, 1081 BT Amsterdam, the Netherlands. E-mail: j.visschedijk@vumc.nl

DOI: $10.1111 / \mathrm{j} .1532-5415.2010 .03036 . x$
A lthough the primary treatment of a hip fracture is mostly surgical, the final functional result also depends on multidisciplinary rehabilitation practices. ${ }^{1,2}$ Several factors have been associated with recovery after a hip fracture, such as age, sex, marital state, residence, premorbid activities of daily living (ADLs), walking ability, cognition, and number of comorbidities. ${ }^{3-5}$ Despite much that is still unknown, the importance of psychological factors has been emphasized. ${ }^{6,7}$ Fear of falling (FoF), in particular, seems to be an important psychological factor, which may have an even greater influence on functional recovery than pain or depression. ${ }^{8}$ FoF also reduces participation in exercises during the rehabilitation process. ${ }^{9,10}$ Functional disabilities caused by FoF may restrict outcomes in the long term, ${ }^{11}$ particularly because FoF is known to result in dependency and poor functioning in older adults. ${ }^{12,13}$

FoF was first used in the context of the postfall syndrome. ${ }^{14}$ Several efforts have been made to operationalize this concept, particularly when measures were being developed. Tinetti describes FoF as "a lasting concern about falling that leads to an individual avoiding activities that he/ she remains capable of performing" and has operationalized FoF as a loss of self-efficacy to perform certain activities without falling. ${ }^{13}$ Others relate FoF to deteriorated postural control. ${ }^{15}$ FoF has often been described more generally as a broader concept of intrinsic fear or worry about falling. ${ }^{16}$

FoF is common in community-based older adults ${ }^{17}$ but may be different in patients after a hip fracture, because these patients have fallen and are suddenly restricted in their activities. In addition, patients with a hip fracture have higher levels of comorbidity and premorbid disability. ${ }^{18,19}$ Hence, the objective of this review was to systematically describe and analyze FoF in patients after hip fracture. The important questions to be addressed were:

Which instruments are used to measure FoF in patients with a hip fracture?

What is the prevalence of FoF among patients with a hip fracture? 
Which factors are associated with FoF after a hip fracture?

Which interventions may reduce FoF after a hip fracture?

A systematic review was conducted to answer these questions. All relevant studies related to FoF in patients with hip fractures were examined in this review.

\section{METHOD}

\section{Data Sources and Search Strategy}

In March 2009 a literature search was conducted using four databases: PubMed (Medline), Embase, PsychINFO, and CINAHL. The Cochrane Library was also consulted. Finally, the reference lists of selected articles were scrutinized for relevant articles.

The databases were searched using controlled terms (e.g., Medical Subject Headings in Medline) and free text words. These were customized to the database. The following search was used most frequently: ((hip fracture*) OR (proximal femur fracture $\left.\left.{ }^{*}\right)\right)$ AND ((fear of fall*) OR (concern of fall*) OR (self-efficacy) OR (fear) OR (psychological factors)).

\section{Study Selection}

All possible studies, retrospective and prospective, were included in the search. Because the majority of hip fractures occur in people aged 65 and older, no age limitation was included. Furthermore, no restriction on the year of publication of the article was made.

The initial search resulted in 819 titles (Figure 1). In PubMed, 362 titles were found, to which 161, 282, and 14 new articles were subsequently added by searching Embase, PsychINFO, and CINAHL, respectively. No additional studies were found in the Cochrane Central Register. Two investigators (WA, JV) screened the titles to find eligible studies. The most important criterion was whether these articles could describe studies related to FoF in patients with hip fractures. Where there was any doubt, the article was included. One hundred fifty-one articles were selected and the abstracts read (WA, JV). Articles were selected when they probably presented a study (not a review) that included FoF

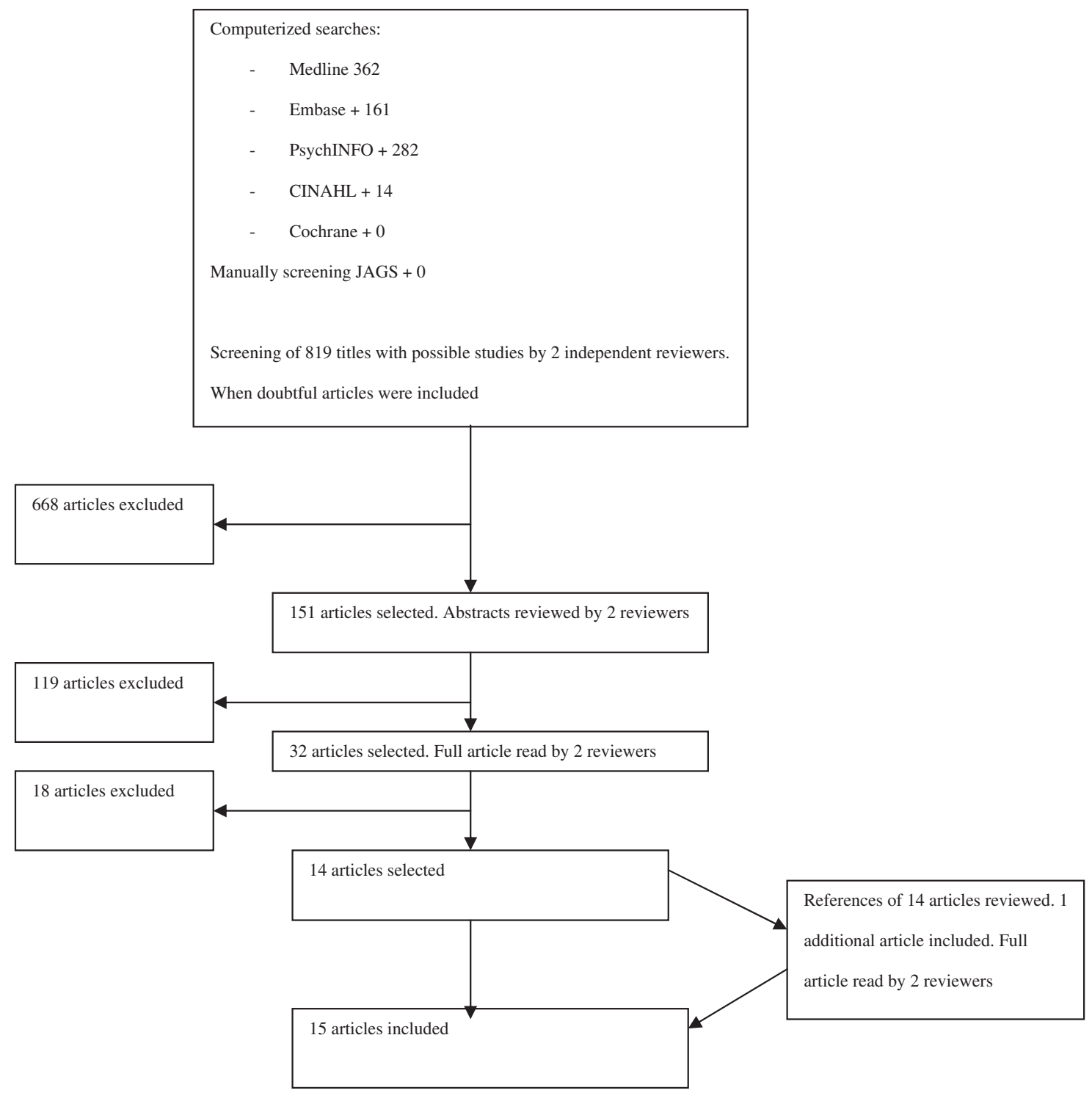

Figure 1. Strategy used for selection of published reports on fear of falling in patients with hip fracture. 
or balance problems in patients with a hip fracture. Furthermore, the full article needed to be available in English, German, French, or Dutch. In addition, the article needed to describe a study and not a comment or personal opinion.

Thirty-two articles met the above-mentioned criteria. Two investigators (WA, JV) read the full articles and assessed their ability to answer the research questions. Qualitative studies and articles in which no analysis for patients with hip fractures was provided were excluded. Fourteen articles were found providing relevant information for the research questions. An additional article was included after reviewing the references.

\section{Data Extraction and Synthesis}

Appraisal tools that the Centre of Evidence-Based Medicine and other institutions provided were used to analyze the quality of the studies. ${ }^{20-23}$ The articles were assessed in particular on validity (Is there a well-defined study question? Is the study design adequate to answer the study question?), importance of results (How great is the likelihood of the results? How precise are the results?), and their applicability to the rehabilitation process (Will the results be helpful for the rehabilitation of our patients? Are the benefits worth the harms and the costs? Do the results fit with other available evidence?). Using this format, studies were further analyzed and evaluated, although it was not possible to make adequate comparisons between the studies and to provide a quality assessment because of the heterogeneity of the studies in terms of design, objectives, variables, and outcome measures. Statistical pooling of data (meta-analysis) was not possible either.

\section{RESULTS}

The 15 studies that were found are summarized in Table 1 .

All studies included measures for FoF. Two studies addressed risk factors for FoF, ${ }^{11,16}$ and one compared different diagnostic measurements. ${ }^{31}$ Eleven studies provided information about the association between FoF and other variables. Four intervention studies could be retrieved in which the effect of an intervention on FoF was assessed. The study features are summarized in Table 1 . Two articles refer to the same group of patients. ${ }^{24,35}$

\section{Which Instruments Are Used to Measure FoF in Patients with a Hip Fracture?}

All studies used at least one instrument to measure FoF. These instruments can be divided into two groups: instruments intended to measure FoF directly and instruments focusing on balance confidence or self-efficacy related to falls. The first group consisted mostly of single items, whereas the second group usually included instruments consisting of several items.

The direct measures for FoF with single items were mostly answers to questions such as "Do you have fear of falling?" or "Are you afraid of falling?" Two instruments were found that measure balance confidence or self-efficacy related to falls: the Activity-related Balance Confidence (ABC) Scale and the Fall Efficacy Scale (FES). The items on the $\mathrm{ABC}$ Scale increase in complexity from the beginning to the end of the instrument. The ABC Scale was used in five studies and the FES in eight. Although these instruments are used for patients with hip fracture, no studies could be found in which the psychometric features of the instruments had been tested for this group of patients.

Studies that had used or compared two or more instruments were of particular interest. One cross-sectional study used the FES (Swedish version; FES(S)) and a direct measure for FoF using a 4-point ordinal scale. ${ }^{31}$ This study, in which patients were assessed approximately 25 days after surgery, found a significant relationship $(P<.001)$ between the two instruments. The less fear a patient felt, the higher their fallrelated efficacy in different activities. Patients who were never or seldom afraid of falling had on average a $40 \%$ higher score on the $\operatorname{FES}(S)$ than patients who reported that they were sometimes or often afraid of falling. A particular advantage of the FES(S) was that it indicated which daily activities the patient perceived to be troublesome, highlighting activities in which the patient might require further training.

Another study found that perceived risk of further falls and worry over further falls were significantly correlated (correlation coefficient $=0.40, P<.001$ ) with each other. ${ }^{16}$ When measured 5 to 8 days after surgery, neither of these measures was significantly associated with the FES, which may indicate that they measure different constructs.

Research also indicated that the FES was more sensitive to change than the ABC Scale. ${ }^{11}$ This is in line with findings from earlier studies in which the FES was used in particular for frail elderly, whereas the ABC Scale, which contains several complex activities, is more often used for relatively healthy community samples. ${ }^{40}$

\section{What Is the Prevalence of FoF in Patients with Hip Fracture?}

No studies were found that specifically focused on the prevalence of FoF in patients with hip fractures. In addition, no studies were found in which FoF was measured systematically over a long period during the rehabilitation process.

Some studies provided useful information about the prevalence of FoF after a hip fracture, although different instruments were used, and evidence-based cutoff points were missing. In some studies, the researchers themselves determined the cutoff point. When FoF was measured within 1 week after surgery on a scale from 1 to $6(1=$ no fear to $6=$ strongest fear), $50 \%(68 / 135)$ of the patients indicated that they were afraid of falling (score of $>3$ ). ${ }^{35}$ Another study, in which FoF was measured on average 25 days after surgery (range 6-80 days), revealed that $65 \%$ $(36 / 55)$ of the patients had FoF sometimes or often. ${ }^{31}$

In an intervention study, FoF was measured on a scale of 1 to 3,3 to 4 weeks after admission to a rehabilitation hospital, after a successive training period of 12 weeks, and 3 months later. ${ }^{30}$ In patients who followed a conventional rehabilitation program, the average FoF was 1.67, 1.55, and 1.78 , respectively. Therefore, only small changes seem to appear over time. Another author indicated an average level of FoF of $2.2(n=149)$ and $2.4(n=166)$ on a scale that ranged from 0 to $4(0=$ no fear, $4=$ strong fear $)$ in two study cohorts 2 months after a hip fracture. ${ }^{37}$

When using the FES(S), the mean score \pm standard deviation $(\mathrm{SD})$ was $5.6 \pm 2.8$ (range $0-10 ; 0=$ no confidence at all, $10=$ full confidence), with higher scores reported for activities such as personal grooming, getting on and off the 
Table 1. Summary of Publications About Fear of Falling After Hip Fracture

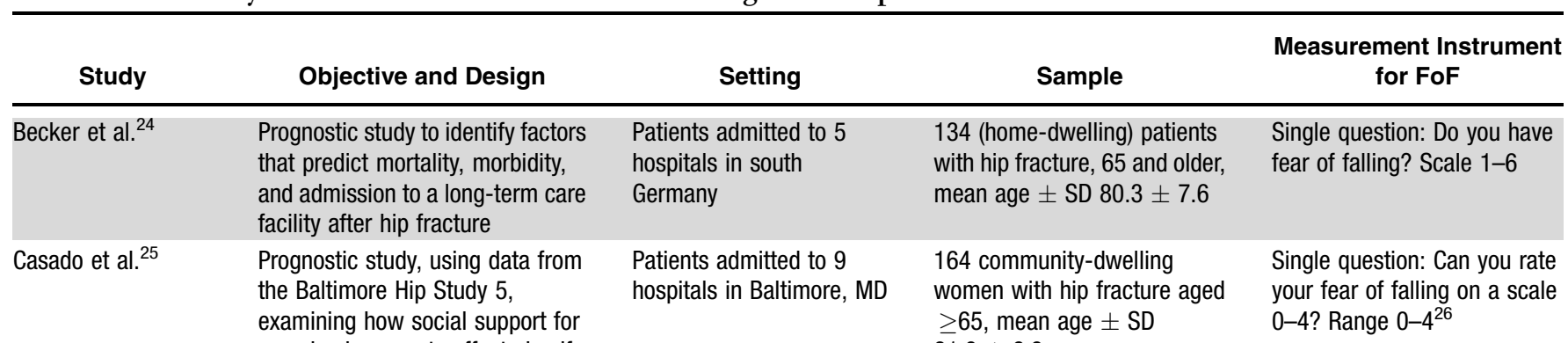
exercise by experts affected selfefficacy, outcome expectations, and exercise behavior

$\begin{array}{ll}\text { Crotty et al. }^{27} & \text { Randomized controlled trial to } \\ & \text { measure effect of intervention } \\ & \text { (home rehabilitation after early } \\ & \text { discharge with therapists visiting } \\ & \text { home focusing on negotiated set } \\ \text { of goals) }\end{array}$

Hauer et al. ${ }^{30} \quad$ Randomized controlled trial to measure effect of intervention (3-month physical training after hip surgery)

\begin{tabular}{|c|c|}
\hline Ingemarsson et al. ${ }^{31}$ & $\begin{array}{l}\text { Diagnostic cross-sectional study } \\
\text { to investigate relationship } \\
\text { between fall-related efficacy and } \\
\text { tests of balance }\end{array}$ \\
\hline Jones et al. ${ }^{33}$ & $\begin{array}{l}\text { Intervention study to assess effect } \\
\text { of community exercise program } \\
\text { (focused on functional stepping } \\
\text { and lower extremity strengthening } \\
\text { exercises) }\end{array}$ \\
\hline Kulmala et al. ${ }^{34}$ & $\begin{array}{l}\text { Cross-sectional study to } \\
\text { investigate association between } \\
\text { self-assessed balance confidence } \\
\text { and functional balance and falls }\end{array}$ \\
\hline McKee et al. ${ }^{16}$ & $\begin{array}{l}\text { Descriptive follow-up study to } \\
\text { determine whether FoF and falls } \\
\text { efficacy contribute to prediction of } \\
\text { health outcomes after hip fracture }\end{array}$ \\
\hline
\end{tabular}

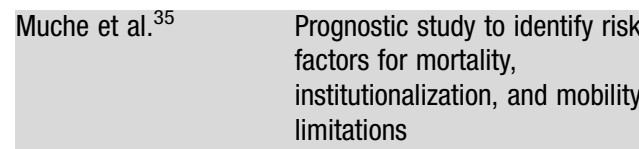

Oude Voshaar et al. ${ }^{8} \quad$ Prospective study to assess effect of factors such as pain, depression, and FoF on functional outcome; part of a randomized controlled trial to prevent and treat depression after hip fracture
Patients admitted to 3 hospitals in Adelaide, Australia
66 patients of aged $>65$ with hip fracture; 34 with accelerated discharge with home-based rehabilitation and 32 allocated to control group with conventional care. Median age (quartiles): intervention group 83.5 (76.6, 85.5); control group 81.6 (78.2, 85.4)

Patients admitted to acute care or inpatient rehabilitation because of hip fracture or hip replacement; Germany

Patients postoperatively cared for at the Geriatric Clinic in Vasa Hospital, Göteborg, Sweden

Patients convalescing in a rehabilitation unit in a teaching hospital, Ontario, Canada

Patients operated on at local hospital in Finland

Patients admitted to the hospital, United Kingdom

28 women with hip fracture aged $>75 ; 15$ in intervention group, 13 in control group, mean age \pm SD $81.3 \pm 3.9$

55 patients operated on for hip fracture, mean age \pm SD $82.3 \pm 6.8$

\section{5 patients aged $\geq 65$ with} hip fracture, the first 17 enrolled in the intervention group, the next 8 controls, mean age \pm SD $80.0 \pm 6.0$ 79 patients operated on with hip fracture aged 60-85, women aged $76.0 \pm 6.2$; men

82 patients with hip fracture aged $\geq 65$, mean age $\pm S D$ aged $73.4 \pm 7.4$ $80.2 \pm 7.3$

Patients admitted to 5 hospitals in Ulm, southern Germany

Patients admitted to one of 4 orthopedic units in Manchester, United Kingdom
135 patients with hip fracture aged $\geq 65$; of 135,15 died in first 6 months so data of 120 patients used for institutionalization and mobility, mean age \pm SD

187 patients with hip fracture aged $\geq 60$, mean age \pm SD $79.8 \pm 8.7$ $80.3 \pm 7.6$
$\mathrm{ABC}$ Scale, 16 items; range $0-100 .^{28}$

FES, 10 items; range $10-110^{29}$
Single question: Are you afraid of falling?

Range $0-3^{15}$
FES - Swedish version, 13 items; range $0-1300^{32}$ Single question: Are you afraid of falling? Range 0-3

ABC Scale, 16 items; range $0-100 \%$ confidence. ${ }^{28}$ FES, 10 items; range $10-100^{29}$

ABC Scale, 16 items; range $16-160^{28}$

Perceived risk of further falls in the next 2 months, 1 item; range 1-6.

Worry over further falls in the next 2 months, 1 item; range $1-6$.

FES, 10 items; range 10-60 29 Single question: Do you have fear of falling? Range 1-6
Modified FES, 14 items; range $0-140^{36}$ 
Table 1. (Contd.)

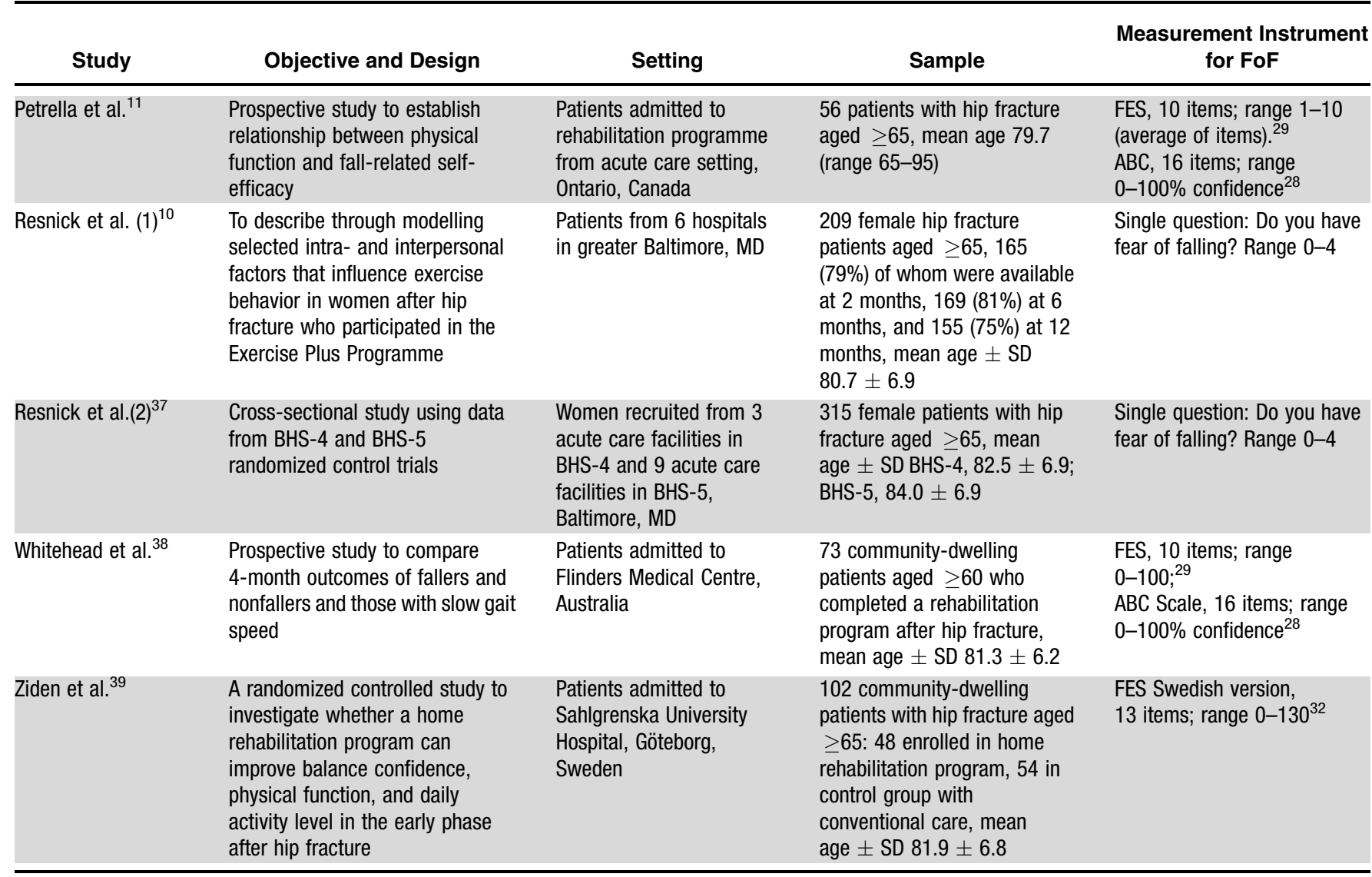

$\mathrm{ABC}=$ Activity-specific Balance Confidence Scale; FES = Falls Efficacy Scale; SD = Standard Deviation; BHS = Baltimore Hip Study.

toilet, getting in and out of a chair, and getting in and out of bed. $^{31}$ The FES(S) was administered 25 days on average after surgical repair of the hip fracture. Another study reported an average score of $69.8 \pm 37.7$ (range 0-140) $(\mathrm{N}=187)$ on the modified FES right after hip fracture. ${ }^{8}$ The wide confidence interval may be due to the heterogeneity of the patients, which was also reflected in wide confidence intervals for depression and pain scales in this study.

\section{Which Factors Are Associated with FoF After a Hip Fracture?}

Associations between FoF and other variables were explored in 11 studies. ${ }^{8,10,11,16,24,25,31,34,35,37,38}$ The relevant variables to which FoF is associated are listed in Table 2.

\section{Premorbid Factors}

One study assessed premorbid factors that may have an influence on FoF. ${ }^{16}$ The information was collected through interviews just after the fracture had occurred. It was found that the FES had a strong association with prefall activity problems and a weaker but significant association with history of falls.

\section{Mortality}

FoF may be a predictor of mortality. This was explored in two longitudinal studies from Germany that used the same population sample. ${ }^{24,35} \mathrm{FoF}$ was the third-best factor after premorbid ADL and sex in this study but the first factor that was possibly modifiable.

\section{Institutionalization}

The above-mentioned studies also found associations, although not significant, between FoF and institutionalization (admission to a nursing home within 6 months after hip fracture). ${ }^{24,35}$

\section{Physical Function, Functional Recovery, and Mobility}

The majority of studies assessed the relationship between FoF and functional outcomes, particularly mobility. ${ }^{8,11,16,24,35,38}$ In two German studies FoF was a predictor for limited outdoor mobility (the capacity of going outdoor without personal assistance). ${ }^{24,35}$

FoF and falls efficacy were assessed as independent variables for the functional limitation dimension of the Functional Limitation Profile (FLP). ${ }^{16}$ Functional limitation at 2 months was associated with perceived risk of further falls $(P=.04)$ and FES score $(P=.005)$ measured approximately 1 week after surgery. These relationships were subsequently examined in multivariate models. With functional limitation as the outcome measure, FES score and perceived risk of further falls did not add significantly to the prediction of variance once length of stay, falls history, and prefall activity problems had been controlled for. 
Table 2. Variables Associated with Fear of Falling (FoF) After Hip Fracture

\begin{tabular}{ccl}
\hline Variable & \multicolumn{1}{c}{ Study } & \multicolumn{1}{c}{ Associated Variable } \\
\hline Prefracture activity & McKee et al. $^{16}$ & $\begin{array}{l}\text { Adapted ADL scale (self-assessed problems } \\
\text { with walking, self-care indoor activities, } \\
\text { outdoor activities) }\end{array}$ \\
History of falls & ${\text { McKee et al. }{ }^{16}}$ & $\begin{array}{l}\text { Fall history (never fallen before; fallen, but not } \\
\text { during last year; fallen in last year) }\end{array}$
\end{tabular}

$\begin{array}{lll}\text { Mortality } & \text { Becker et al. }^{24} & \text { Mortality within } 6 \text { months after surgery } \\ & \text { Muche et al. }{ }^{35} & \text { Mortality within } 6 \text { months after surgery }\end{array}$

Institutionalization Becker et al. ${ }^{24} \quad$ Living in nursing home 6 months after surgery

Muche et al. ${ }^{35}$

Living in nursing home 6 months after surgery

Physical function functional recovery, balance, mobility

Ingemarsson et al. ${ }^{31}$

Functional reach; balance tests on platform

McKee et al. ${ }^{16} \quad$ Functional recovery from injury: physical limitation dimension of the FLP

Muche $\mathrm{R}$ et al. ${ }^{35}$

Ability to go outdoors without help of others

Oude Voshaar et al. ${ }^{8} \quad$ TUG; gait speed, FR, activity subscale of selfreport Sickness Impact Profile questionnaire

$\begin{array}{ll}\text { Petrella et al. } & { }^{11} \quad \begin{array}{l}\text { Physical function: Functional Independence } \\ \text { Measure }\end{array}\end{array}$

Whitehead et al. ${ }^{38} \quad 10-\mathrm{m}$ walk test for gait speed

$\begin{array}{lll}\text { Exercise } & \text { Casado et al. }{ }^{25} & \text { Outcome Expectations for Exercise Scale } \\ & \text { Self-Efficacy for Exercise Scale } \\ & {\text { Resnick et al. }{ }^{10}} & \text { Social Support for Exercise Scale } \\ & \text { Self-Efficacy for Exercise Scale } \\ & \text { Outcome Expectations for Exercise Scale } \\ & \text { Stage of Change Questionnaire } \\ & \text { Exercise Time }\end{array}$

Resnick et al. ${ }^{37} \quad$ Self-Efficacy for Exercise scale Outcome Expectations for Exercise scale Yale Physical Activity Survey
FES associated with prefall activity problems $(P<0.001)$. Association between ADL scale and "worry over further falls in next two months" and "perceived risk of further falls in the next two months" not significant.

FES was associated with fall history $(P<.05)$. Worry over further falls in next 2 months was associated with fall history $(P<.001)$. Association between fall history and "perceived risk of further falls in the next two months was not significant.

Multivariate logistic model: FoF OR $=4.22$ for mortality, $95 \%$ $\mathrm{Cl}=0.80-4.80$.

Percentage of patients who died was $17.7 \%$ for patients with strong $\mathrm{FoF}$ and $4.5 \%$ for patients without $(P=.02)$

Multivariate logistic model: $\mathrm{FoF}$ for institutionalization: $\mathrm{OR}=2.23,95 \% \mathrm{Cl}=0.79-6.27$.

Percentage of patients who were institutionalized was $31.1 \%$ for patients with strong $\mathrm{FoF}$ and $17.2 \%$ for patients without $\mathrm{FoF}$ $(P=.06)$.

Multivariate logistic model: FoF for loss of mobility $\mathrm{OR}=1.96$, $95 \% \mathrm{Cl}=0.80-4.80$.

Significant relationship between subjective ability (FES) and objectively measured balance (FR) $(P<.001)$; only a few significant correlations between balance tests on platform and FES(S) and FR.

Physical limitation dimension at 2 months was associated with FES score $(P=.005)$; physical limitation dimension at 2 months was associated with perceived risk of further falls $(P=.05)$; physical limitation dimension at 2 months was not significantly associated with worry over further falls.

Percentage of patients with mobility limitations was $37.5 \%$ for patients with strong FoF and $18.8 \%$ for patients without FoF $(P=.02)$.

FoF to predict TUG at 6 months: baseline $\mathrm{OR}=0.89(P=.04)$ and after 6 weeks $\mathrm{OR}=0.75(P<.001)$

FoF to predict gait speed at 6 months: baseline $\mathrm{OR}=0.93$ (not significant) and after 6 weeks $\mathrm{OR}=0.73(P<.001)$.

FoF to predict FR at 6 months: baseline $\mathrm{OR}=1.06$ (not significant) and after 6 weeks $\mathrm{OR}=1.32(P=.006)$. FoF to predict Sickness Impact Profile at 6 months: baseline $\mathrm{OR}=0.92(P=.11)$ and after 6 weeks $\mathrm{OR}=0.70(P<.001)$

No correlation was found between changes in the fall-related self-efficacy measures and the Functional Independence Measure

Those with slower gait speed had lower self-efficacy (FES and $A B C)$. Patients with normal gait: mean FES $71.3 \pm 22.9$, mean $\mathrm{ABC} 45.6 \pm 21.0$; patients with slow gait: mean FES $78.6 \pm 33.8$, mean ABC $75.5 \pm 16.6$.

Model indicated significant path between FoF and outcome expectations for exercise.

At 2 months, FoF was not significantly related to any of the variables mentioned (in the table). At 6 months, FoF was related to outcome expectations for exercise (path coefficient -0.23 ; $P<.001)$ and indirectly related to exercise time.

At 12 months, participants with less FoF had strong self-efficacy expectations (path coefficient $-0.25, P<.001$ ). FoF related also to outcome expectations (path coefficient $-0.23, P<.001$ ). Through these, FoF was related to time spent in exercise.

The participants reported some FoF; however, no significant relation between FoF and self-efficacy expectations and exercise behavior. 
Table 2. (Contd.)

\begin{tabular}{|c|c|c|c|}
\hline Variable & Study & Associated Variable & Association \\
\hline \multirow[t]{2}{*}{ Falls } & Kulmala et al. ${ }^{34}$ & $\begin{array}{l}\text { Berg Balance Scale for functional balance } \\
\text { Self-reported falls during previous } 6 \text { months } \\
\text { Falls vs no falls } \\
\text { Recurrent falls vs occasional or no falls } \\
\text { Indoor falls vs no indoor falls Outdoor falls vs } \\
\text { no outdoor falls }\end{array}$ & $\begin{array}{l}\text { Lower } A B C \text { score was associated with recurrent falling and lower } \\
\text { BBS score. } \\
\text { Mean } A B C \text { for no recurrent falls was } 97 \pm 31 \text {, versus } 68 \pm 51 \\
\text { for recurrent falls. Lower } A B C \text { scores were also related to indoor } \\
\text { falls. Mean } A B C \text { score for no indoor falls was } 100 \pm 32 \text {, versus } \\
72 \pm 35 \text { for indoor falls. Patients with outdoor falls did not differ } \\
\text { from those with no outdoor falls in } A B C \text { scores. }\end{array}$ \\
\hline & Whitehead et al. ${ }^{38}$ & Fall history & $\begin{array}{l}\text { Those who had fallen had lower fall self-efficacy. Fallers: FES } \\
\text { score } 61.7 \pm 22.6, A B C \text { score } 33.4 \pm 20.1 \text {; nonfallers: FES } \\
\text { score } 73.5 \pm 26.2, A B C \text { score } 53.5 \pm 23.0\end{array}$ \\
\hline
\end{tabular}

$\mathrm{ABC}=$ Activity-specific Balance Confidence Scale; $\mathrm{ADL}=$ activity of daily living; $\mathrm{BBS}=$ Berg Balance Scale; $\mathrm{CI}=$ confidence interval; FES = Falls Efficacy Scale; FLP = Functional Limitation Profile; FR = functional reach; $\mathrm{OR}=$ odds ratio; $\mathrm{SD}=$ standard deviation; TUG = Timed Up and Go Test.

The relationship between FoF and functional outcomes was strongly established in another longitudinal study. ${ }^{8}$ In the final multivariate model, cognitive functioning and FoF (Modified FES) assessed 6 weeks after surgery consistently predicted functional recovery at 6 months, measured using the Get Up and Go Test, gait speed, and functional reach. Also, the overall multivariate models including all psychological variables (cognition, pain, depression) consistently included FoF at 6 weeks as the most significant predictor after correction for other factors such as age and level of premorbid functioning.

Another study found no relationship between changes in physical functioning (Functional Independence Measure, ADL, mobility) during a rehabilitation program and changes in fall-related self efficacy (FES and ABC). ${ }^{11} \mathrm{An}$ other author compared groups with different functional outcomes (those with normal walking speed vs those with low walking speed: slower than 2 standard deviations (SDs) below the mean in a $10-\mathrm{m}$ timed walking test). ${ }^{38}$ The mean of the FES and the ABC 4 months after surgery were significantly lower for slow walkers than normal walkers.

\section{Exercise}

Data from two cohorts in the Baltimore Hip Studies (BHS-4 and BHS-5), in which an intervention (Exercise Plus Programme) was tested, were also used to assess FoF. ${ }^{37}$ When women were tested at 2 months, no significant relationships between FoF and participation in exercises could be demonstrated. In another study, using data from the Baltimore Hip Studies, data were collected at 2, 6, and 12 months, and structural equation models including FoF were tested. ${ }^{10}$ Although FoF at 2 months was not significantly related, at 6 months it was related to exercise time. In addition, at 12 months, those with less FoF spent more time in exercise. A model developed to analyze data from the BHS- 5 indicated an association between FoF and exercise. ${ }^{25}$

\section{Falls}

Three studies focused on the relationship between FoF and falls. ${ }^{16,34,38}$ In a cross-sectional study, 79 patients were assessed who had undergone surgery for hip fracture 6 months to 7 years before. ${ }^{34} \mathrm{~A}$ lower $\mathrm{ABC}$ score was associated with recurrent falling and a lower Berg Balance Score. Participants with indoor falls had lower ABC scores, but no difference in $\mathrm{ABC}$ score was found between outdoor falls and no outdoor falls. Another author found that "no history of falls" 2 months after hospital discharge was negatively associated with worry over further falls $(P=.005)$ and positively with FES score $(P<.05) .{ }^{16}$

Finally the association between FoF and falls was confirmed when differences between groups of fallers and nonfallers were studied. Those who had fallen in the 4 months after hip fracture had significantly lower FES and ABC scores at the 4-month follow-up. ${ }^{38}$

\section{Which Interventions May Reduce FoF After a Hip Fracture?}

The effect of an intervention on FoF was assessed in four studies, ${ }^{27,30,33,39}$ three of which were randomized controlled trials. ${ }^{27,30,39}$ Patients with severe comorbidity or cognitive disorders and patients who were not expected to return home were mostly excluded.

One study ${ }^{27}$ evaluated a home-based rehabilitation program with early discharge. After discharge, therapists visited patients at home and negotiated a set of targets. Patients followed this program on average for 28 days. As a result of strict inclusion criteria, only 66 of 188 patients were included. The study found that the mean FES score at 4 months was significantly better for the intervention group. The mean ABC score of patients was not significantly different between the intervention and control group.

Another study ${ }^{30}$ investigated a 12 -week program of ambulatory training that started immediately after discharge 
from the hospital. The program included intensive training of relevant muscle groups and functional training to enhance balance. Measurements were taken 3 to 4 weeks after admission to the hospital, at the end of the training period, and 3 months later. Although there was a clear improvement in FoF, it was not significant. The mean FoF score in the intervention group decreased from $1.50 \pm 0.71$ to $0.78 \pm 0.83$ at the end of the training period; 3 months later FoF was $1.00 \pm 0.92$. For the control group, only a small decrease was found-from $1.67 \pm 1.0$ to $1.55 \pm 0.88$ - whereas after 3 months, FoF increased to $1.78 \pm 0.67$.

A community exercise program focusing on functional stepping and lower extremity strengthening exercises was evaluated after a 4 -month intervention period. ${ }^{33}$ The first 17 patients were enrolled in the intervention group, and the next 10 consecutive patients were controls. The ABC score increased in the intervention group from $76.6 \pm 21.8$ to $90.1 \pm 10.1$, compared with an increase in the control group from $80.8 \pm 19.1$ to $94.3 \pm 6.1$. FES increased in the intervention group from $83.9 \pm 15.0$ to $93.6 \pm 6.6$, compared with an increase in the control group from $89.1 \pm 10.8$ to $94.4 \pm 6.7$. The differences were not significant between the intervention and control groups.

In a study of a home rehabilitation program that had a maximum period of 3 weeks after discharge and was aimed to improve balance confidence, physical function, and ADLs, the intervention group reported significantly higher confidence in performing daily activities, as measured by the FES. ${ }^{39}$ The intervention group had a larger increase than controls in balance confidence on stairs and instrumental activities 1 month after discharge according to the FES. The improvements in the means of the total score for the intervention and control groups were 30.6 and 13.5 , respectively $(P<.001)$; the improvements in the means of the stair climbing item for the intervention and control group were 3.3 and 0.6 , respectively $(P=.002)$; and the improvements in the means of the instrumental ADL items of the FES for the intervention and control groups were 19.7 and 7.1, respectively $(P<.001)$.

\section{DISCUSSION}

In this review, 15 studies related to FoF in patients with hip fracture were evaluated. The studies provided information concerning measuring FoF, the prevalence of FoF, associations between $\mathrm{FoF}$ and other variables, and interventions to improve FoF.

Measurement instruments can be divided into two groups: those that directly assess FoF using a single question and those that particularly relate to keeping balance or selfefficacy in not falling during certain activities, such as the $\mathrm{ABC}$ Scale and FES. The ABC Scale comprises many complex activities and has a greater responsiveness for people with a higher degree of functioning than patients after hip fracture. The FES was used in several modifications, sometimes focusing on the confidence someone has in not falling when doing an activity and sometimes explicitly on the fear someone has about losing balance and falling during an activity. Modified versions of the FES have been developed because the FES probably has a ceiling effect ${ }^{39}$ (e.g., the international version (FES-I), to which more-difficult and social activities have been added). For frail elderly patients after hip fracture, the FES-I, similar to the ABC, may comprise activities that are too complex, and the ceiling effect may be less relevant. The FES(S) may be more suitable for patients with hip fracture, because it focuses on basic ADLs, which are relevant for patients with moderate to low functional ability. ${ }^{32}$

No studies were found that assessed the psychometric features of these instruments for patients with a hip fracture. A systematic review of measurement instruments for the psychological outcomes of falling evaluated the available instruments for $\mathrm{FoF}^{40}$ Most of the instruments found in the current review can also be found in that study, which identified the same main categories (instruments that intend to measure FoF directly and those that focus on fall-related efficacy and confidence, indicating that these are different constructs). In a few studies in which single-item instruments and FES instruments were included, a correlation was found. It is likely that someone who has FoF also has less confidence in performing certain activities that require balance. Exactly how these constructs interact with each other requires further research. In addition, other factors such as coping behavior, motivation, and outcome expectations may influence self-efficacy to execute certain activities. That study concluded that "the majority of research reporting psychometric properties has focused on selfefficacy measures. These instruments may prove superior to others because of the strong and well-researched theoretical base." Because almost all research has focused on healthy community-dwelling older adults, evidence is lacking as to whether this statement can be extrapolated to all patients with hip fracture.

No studies were found that consistently assessed the prevalence of FoF after hip fracture over a long time period. Most studies used different instruments, and the period between hip fracture and measurement varied substantially. Therefore, it is difficult to compare these findings, because FoF may not be stable over the rehabilitation period. Another limitation is that all studies excluded patients with cognitive and severe medical disorders, which may give selection bias. It is possible that particularly patients with cognitive and severe comorbidity suffer more often from FoF. A literature review reported that, in community-dwelling older adults, the prevalence of FoF varies between $21 \%$ and $85 \% .{ }^{17}$ The findings of the studies in this review are within these limits.

Many factors have been associated with FoF in community-based older adults. ${ }^{17}$ Some of these were also found in the current review. Because most of the studies were cross-sectional, the causality between these factors remains unclear. Only premorbid activity and history of falls were shown to be risk factors for FoF after a hip fracture. ${ }^{16}$ Furthermore, this review reveals that FoF is a predictor of important outcomes for the rehabilitation process, such as mobility, mortality, and institutionalization. Further research is needed to establish whether causal relationships exist with other factors. FoF was related to falling, but not with outdoor falls. ${ }^{34}$ It is possible that lack of FoF is a risk factor for outdoor falls because patients with a low ABC score are more reluctant to walk outside and are more careful. Patients with severe FoF may reduce their activities and spend more time indoors. FoF may work protectively for these older adults, although the study may have some 
flaws due to recall bias (for falls) and because only a minority of the potential participants consented to participate in the study.

The finding that FoF may be related to exercise is particularly important. ${ }^{25}$ It may imply that FoF has to be addressed throughout the rehabilitation process, because exercise improves health outcomes. ${ }^{2}$ One study found that the effect of FoF seemed to be strongest 12 months after fracture rather than in the more-immediate postfracture period, ${ }^{10}$ which "suggests that ongoing efforts might be made to address the FoF well after their initial fracture." In addition, it has been speculated that "the level of fear of falling during rehabilitation is a more important predictor for functional outcome than fear of falling directly after surgery by excluding patients who easily overcome their initial anxiety and including those who become aware of their fear during rehabilitation." ${ }^{8}$ More research is required to establish the precise (causal) relationship between FoF and important outcomes.

Intervention studies have revealed that FoF can be modified, ${ }^{27,39}$ but the studies have to be interpreted with care, because they included only relatively healthy patients, possibly causing a selection bias. It is possible that patients with more-severe medical and cognitive disorders have less favorable results because they are less trainable and motivated. In one study, ${ }^{30} 14$ of the 28 patients included underwent a total hip replacement, which is a less common procedure for hip fracture and makes it cumbersome to generalize these results to other populations. In addition, sample sizes of the studies were small, and the follow-up periods were mostly short. In one study, the small sample may have caused the association not to be significant. ${ }^{30} \mathrm{In}$ another study, the high number of nonconsenters and the strict inclusion criteria may have caused selection bias. ${ }^{33}$ Furthermore, the control and intervention groups may not have been comparable from the start, as indicated by the differences between the groups in relation to the FES scores at baseline. In another study, the difference in effect of the intervention on FoF may be even stronger, with six patients in the home-based rehabilitation program not receiving it (intention-to-treat principle) and several patients in the conventional care group receiving other types of treatment after discharge. ${ }^{39}$ Because the intervention had only 1 month of follow-up, it is not clear whether these improvements will be sustained.

Over the past years, several interventions, particularly for community-based older adults, have been developed to reduce FoF. $^{41,42}$ Different programs have been implemented, some focusing more on exercise (balance training, walking, tai chi) and others more on education (discussions about risk of falling, adequate feeding habits, and being active). Whether such programs are also useful for patients after hip fracture is largely unknown and requires further research.

A major limitation of this review is the absence of a substantial number of prospective studies. Most studies were cross-sectional, which makes it impossible to describe the severity of FoF during the rehabilitation process and to find causal relationships between FoF and relevant outcomes. Prospective studies are necessary to bring more clarity. Another limitation relates to the inclusion of predominantly relatively healthy older adults in the studies. It makes generalization of results to the whole population of people with hip fracture cumbersome, because a high proportion of patients with hip fracture suffer from chronic diseases, both physical and mental in nature. ${ }^{18,19}$ Finally, the studies included in this review had a wide variety of designs and methodologies, addressing FoF in different modalities. This made comparison between studies and adequate rating not suitable.

This review has shown that FoF in patients with hip fracture is common, although adequate instruments still have to be validated for this specific group. FoF is associated with several negative rehabilitation outcomes. Knowledge about risk factors of FoF, prevalence over a longer time period, and the exact causal relationship with important health outcomes are still obscure. This information is needed to improve the outcomes of rehabilitation after hip fracture, particularly for patients who also have additional cognitive and medical disorders. Based on this knowledge, adequate interventions can be developed that may reduce FoF and improve outcomes of rehabilitation after a hip fracture.

\section{ACKNOWLEDGMENTS}

Conflict of Interest: The editor in chief has reviewed the conflict of interest checklist provided by the authors and has determined that the authors have no financial or any other kind of personal conflicts with this paper.

Author Contributions: All authors participated in the study design. Data collection: WA and JV. Data analysis and interpretation: WA, JV, and RB. Drafting of the manuscript: JV and WA. All authors assisted with revisions to the manuscript and approved the final version.

Sponsor's Role: None.

\section{REFERENCES}

1. Shabat S. Hip fractures in elderly patients - perspectives towards the future. Disabil Rehabil 2005;27:1039-1040.

2. Chudyk AM, Jutai JW, Petrella RJ et al. Systematic review of hip fracture rehabilitation practices in the elderly. Arch Phys Med Rehabil 2009;90:246262.

3. Balen VR, Steyerberg EW, Polder JJ et al. Hip fracture in elderly patients. Outcomes for function, quality of life and type of residence. Clin Orthop Rel Res 2001;390:232-243.

4. Michel J, Hoffmeyer P, Klopfenstein et al. Prognosis of functional recovery 1 year after hip fracture: Typical patient profiles through cluster analysis. J Gerontol A Biol Sci Med Sci 2000;55A:M508-M515.

5. Osnes EK, Lofthus CM, Meyer HE et al. Consequences of hip fracture on activities of daily life and residential needs. Osteoporos Int 2004;15:567-574.

6. Mossey J, Mutran E, Knott K et al. Determinants of recovery 12 months after hip fracture; the importance of psychosocial factors. Am J Public Health 1989;79:279-286.

7. Fortinsky RH, Bohannon RW, Litt MD et al. Rehabilitation therapy selfefficacy and functional recovery after hip fracture. Int J Rehabil Res 2002; 25:241-246.

8. Oude Voshaar RC, Banerjee S, Horan M et al. Fear of falling more important than pain and depression for functional recovery after surgery for hip fracture in older people. Pschol Med 2006;36:1635-1645.

9. Lees FD, Clarck PG, Nigg RN et al. Barriers to exercise behavior among older adults: A focus group discussion study. J Aging Phys Act 2005;13:23-33.

10. Resnick B, Orwig D, D'Adamo C et al. Factors that influence exercise activity among women post hip fracture participating in the Exercise Plus Program. Clin Interv Aging 2007;2:413-427.

11. Petrella RJ, Payne M, Meyers A et al. Physical function and the fear of falling after hip fracture rehabilitation in the elderly. Am J Phys Med Rehabil 2000;79:154-160. 
12. Wijlhuizen GJ, Chorus AM, Hopman-Rock Fragility, fear of falling, physical activity and falls among older persons: Some theoretical considerations to interpret mediation. Prev Med 2008;46A:612-614.

13. Tinetti ME, Powell L. Fear of falling and low self-efficacy: A case of dependence in elderly persons. J Gerontol 1993;48:35-38.

14. Legters K. Fear of falling. Phys Ther 2002;82:264-272.

15. Maki BE, Holliday PJ, Topper AK. Fear of falling and postural performance in the elderly. J Gerontol A Biol Sci Med Sci 1991;46A:M123-M131.

16. Mckee KJ, Orbell S, Austin CA et al. Fear of falling, falls efficacy, and health outcomes in older people following hip fracture. Disabil Rehabil 2002;24:327-333.

17. Scheffer AC, Schuurmans MJ, Dijk van N et al. Fear of falling; measurement strategy, prevalence, risk factors and consequences among older persons. Age Ageing 2008;37:19-24.

18. de Luise C, Brimacombe M, Pederson et al. Comorbidity and mortality following hip fracture: A population-based cohort study. Aging Clin Exp Res 2008;20:412-418

19. Liebson CL, Tosteson ANA, Gabriel SE et al. Mortality, disability, and nursing home use for persons with and without hip fracture: A population-based study. J Am Geriatr Soc 2002;50:1644-1650.

20. Public Health Resource Unit [on-line]. Available at http://www.phru.nhs.uk Accessed on May 8, 2009

21. Centre for Evidence Based Medicine. Critical Appraisal Tools 2006 [on-line]. Available at http://www.cebm.net Accessed on May 8, 2009.

22. Centre for Health Evidence. Users' Guides to Evidence-Based Practice [online]. Available at http://www.cche.net Accessed on May 8, 2009.

23. Evidence Based Medicine Toolkit. University of Alberta [on-line]. Available at http://www.ebm.ualberta.ca Accessed on May 8, 2009.

24. Becker C, Gebhard F, Fleischer S et al. Prediction of mortality, mobility and admission to long-term care after hip fractures. Unfallchirurg 2003;106: 32-38.

25. Casado BL, Resnick B, Zimmerman $S$ et al. Social support for exercise by experts in older women post-hip fracture. J Women Aging 2009;21:48-62.

26. Resnick G, Daly MP. Predictors of functional ability in geriatric rehabilitation patients. Rehabil Nurs 1998;23:21-29.

27. Crotty M, Whitehead CH, Gray S et al. Early discharge and home rehabilitation after hip fracture achieves functional improvements: A randomized controlled trial. Clin Rehabil 2002;16:406-413.
28. Powell LE, Meyers The Activities-specific Balance Confidence (ABC) Scale. J Gerontol A Biol Sci Med Sci 1995;50A:M28-M34.

29. Tinetti ME, Richman D, Powell L. Falls efficacy as an measure of fear of falling. J Gerontol 1990;45:239-243.

30. Hauer K, Specht $\mathrm{N}$, Schuler $\mathrm{M}$ et al. Intensive physical training in geriatric patients after severe falls and hip surgery. Age Ageing 2002;31:49-57.

31. Ingemarsson AH, Frändin K, Hellström $\mathrm{K}$ et al. Balance function and fallrelated efficacy in patients with a newly operated hip fracture. Clin Rehabil 2000;14:497-505

32. Hellström K, Lindmark B. Fear of falling in patients with a stroke: A reliability study. Clin Rehabil 1999;13:509-517.

33. Jones GR, Jakobi JM, Taylor AW et al. Community exercise programme for older adults recovering from hip fracture. J Aging Phys Act 2006;14:439-455.

34. Kumala J, Sihvonen S, Kallinen $M$ et al. Balance confidence and functional balance in relation to falls in older persons with hip fracture history. J Geriatr Phys Ther 2007;30:114-120.

35. Muche R, Eichner, Gebhard F et al. Risikofaktoren und prognosemöglichkeiten fur mortalität und soziofunktionelle Einschränkungen bei Älteren nach proximalen Femurfrakturen. Euro J Ger 2003;5:187-194.

36. Hill KD, Schwarz JA, Kalogeropoulos AJ et al. Fear of falling revisited. Arch Phys Med Rehabil 1996;77:1025-1029.

37. Resnick B, Orwig D, Hawkes W et al. The relationship between psychological state and exercise behavior of older women 2 months after hip fracture. Rehabil Nurs 2007;32:139-147.

38. Whitehead C, Miller M, Crotty M. Falls in community-dwelling older persons following hip fracture: Impact on self-efficacy, balance and handicaps. Clin Rehabil 2003;17:899-906.

39. Ziden L, Frändin K, Kreuter M. Home rehabilitation after hip fracture. A randomized controlled study on balance confidence, physical function and every activities. Clin Rehabil 2008;22:1019-1033.

40. Jorstad E, Hauer K, Becker C et al. Measuring the psychological outcomes of falling: A systematic review. J Am Geriatr Soc 2005;53:501-510.

41. Jung D, Lee Y, Lee SM. A meta-analysis of fear of falling treatment programmes for the elderly. West J Nurs Res 2009;31:6-16.

42. Zijlstra GA, van Haastregt JC, van Rossum E et al. Interventions to reduce fear of falling in community-living older people: A systematic review. J Am Geriatr Soc 2007;55:603-615. 